\title{
Medicare Coverage of Acupuncture for Chronic Low Back Pain: Does It Move the Needle on the Opioid Crisis?
}

\author{
Kevin T. Liou, $M D^{\top} \odot$, Deborah Korenstein, $M^{2}$, and Jun J. Mao, MD, MSCE \\ 'Bendheim Integrative Medicine Service, Department of Medicine, Memorial Sloan Kettering Cancer Center, New York, NY, USA; ${ }^{2}$ Center for \\ Health Policy and Outcomes, Memorial Sloan Kettering Cancer Center, New York, NY, USA.
}

$\mathrm{J}$ Gen Intern Med 36(2):527-9

DOI: $10.1007 / \mathrm{s} 11606-020-05871-6$

(c) Society of General Internal Medicine 2020

$\mathrm{O}$ n January 21, 2020, in response to the opioid crisis, the Centers for Medicare and Medicaid Services (CMS) announced a decision to cover acupuncture for Medicare patients with chronic low back pain. ${ }^{1}$ Among older adults, chronic pain is prevalent and often debilitating, and is the most common indication for opioid therapy despite limited evidence of efficacy. Opioids carry significant risk in this population, with the number of opioid-related deaths increasing more than sixfold among Americans age 65 and older between 2001 and 2016. ${ }^{2}$ The CMS decision represents a promising step towards expanding non-pharmacological treatment options and reducing reliance on opioids; however, further action is needed to translate Medicare coverage of acupuncture into equitable and sustainable access. This Viewpoint considers key barriers to acupuncture dissemination and implementation and recommends strategies for addressing these obstacles. The new Medicare acupuncture policy provides a lens to explore the broader challenges ahead in pain management as policymakers seek to expand non-pharmacological options in the aftermath of the opioid crisis.

\section{ACUPUNCTURE AND THE NEW MEDICARE POLICY}

Derived from Traditional Chinese Medicine, acupuncture encompasses a diverse array of therapeutic modalities that generally involve insertion, manipulation, and/or stimulation of needles at specific points on the body. Although its comparative effectiveness against other pain management modalities has not been rigorously studied, acupuncture has demonstrated efficacy, safety, and cost-effectiveness for the treatment of chronic low back pain. ${ }^{3}$ Based on this evidence, CMS will now cover up to 12 acupuncture treatments for chronic low back pain, followed by an additional 8 treatments if patients

Received March 2, 2020

Accepted April 22, 2020

Published online May 6, 2020 demonstrate clinical improvement. Acupuncture delivered by appropriately credentialed physicians and advanced practice providers (e.g., physician assistants, nurse practitioners) will be covered; however, CMS does not recognize licensed acupuncturists as Medicare providers, so their treatments will only be covered if delivered under appropriate supervision from a Medicare provider.

\section{BARRIERS TO DISSEMINATION AND IMPLEMENTATION OF ACUPUNCTURE}

Although Medicare coverage of acupuncture will help to expand access, limited acupuncture availability remains a significant barrier that may diminish the impact of the CMS policy, particularly in communities hit hardest by the opioid crisis. In the USA, there are an estimated 38,000 licensed acupuncturists; however, nearly $50 \%$ are located in New York, California, and Florida, which have relatively low opioid prescription rates among Medicare patients compared with other states. ${ }^{4,5}$ By contrast, states with high opioid prescription rates and rising trends in opioid-related deaths, such as Ohio, Michigan, and Kentucky, have fewer than 2 acupuncturists per 100,000 people, highlighting a potential gap between availability and demonstrated need for acupuncture services. ${ }^{4,5}$ Acupuncture clinics also tend to be concentrated in urban, upper-income neighborhoods, raising concerns about access in rural or poor communities. ${ }^{6}$ Most acupuncturists operate private practices without supervision from Medicare providers and thus would not be eligible for coverage under the new CMS policy.

To date, approximately 10,000 physicians have received acupuncture training, but their clinical volume, practice models, and geographic distribution remain largely unknown. The majority of Medicare providers lack familiarity with acupuncture and may not be appropriately equipped to provide acupuncturist supervision required for Medicare coverage. These knowledge gaps also prevent providers from effectively counseling and educating patients on the role of acupuncture in pain management. Few patients treated by acupuncturists are referred by other healthcare providers.

These barriers - limited availability, lack of provider knowledge, and endorsement - all contribute to reluctance among patients to seek acupuncture. Our research suggests 
that a sizeable proportion of patients are not willing to use acupuncture for pain management, even if treatments are covered by insurance. ${ }^{7}$ Lack of knowledge or awareness about acupuncture remains a major barrier that may be particularly salient among those at higher risk of opioid-related mortality. For example, patients with low educational attainment are less willing to seek acupuncture and more likely to overdose on opioids. ${ }^{7}$ Similarly, black patients experience significant disparities in pain management, including a recent trend of higher increases in opioid-related deaths compared with white patients; however, black patients are typically less open to acupuncture. ${ }^{7}$ Practical considerations may also deter patients from engaging in acupuncture. A full treatment course, typically at least 6-10 weekly or biweekly in-person treatments, requires significant investment of time and frequent traveling that may be infeasible for an older Medicare population with greater comorbidity and physical or cognitive impairments, particularly those living in rural areas. Despite concerns about polypharmacy among older patients, it is often more convenient to take an analgesic than to grapple with these challenges.

\section{STRATEGIES FOR INTEGRATION OF ACUPUNCTURE INTO PAIN MANAGEMENT}

To maximize the impact of the new Medicare policy on the opioid crisis, thoughtful dissemination and implementation strategies must be adopted at multiple levels. Increasing Medicare workforce capacity to deliver acupuncture is an important next step. A recent nationwide initiative has paved the way for this work. In 2013, the Department of Defense established Acupuncture Training Across Clinical Settings (ATACS) to address unmet pain management needs among veterans. ${ }^{8}$ To promote scalability and ease of administration, ATACS taught Veterans Administration healthcare providers how to deliver an adapted, standardized acupuncture protocol involving only points in the ears. ${ }^{8}$ Within three years, ATACS successfully trained over 2700 providers, and a randomized controlled trial evaluating the auricular acupuncture protocol's effectiveness is ongoing. For providers who lack the time or interest to undergo intensive acupuncture training, brief and focused educational interventions may be necessary to address knowledge gaps and to inform how to effectively counsel patients about acupuncture. Approximately half of American medical schools lack curricular content on acupuncture and thus may represent promising intervention targets for dissemination efforts.

The supervision requirement under the new CMS policy offers another mechanism to increase availability through interprofessional collaboration between licensed acupuncturists and Medicare providers. Payers and funders should incentivize advancements in team science to determine the best way to coordinate care and integrate providers from different medical paradigms into a cohesive pain management care model. Such work would have broad applicability as multi- disciplinary approaches are increasingly adopted in pain management.

These provider-focused efforts must be complemented by patient education interventions involving strategic, targeted dissemination of information to those who lack awareness about acupuncture. To prevent widening sociodemographic disparities, acupuncture implementation must also examine care delivery formats that overcome barriers among minority and economically disadvantaged populations. For example, community-based, group delivery models have demonstrated feasibility and acceptability in diverse, medically underserved populations, with studies of effectiveness ongoing; these findings can inform future initiatives to promote more equitable access to acupuncture. The physical and cognitive limitations of an aging Medicare population must also be considered. Potential strategies to promote accessibility include coordination with transportation services to provide rides to treatments; partnerships with nursing homes or assisted living facilities to establish in-house acupuncture clinics; or adaptations of the home visit care model to deliver treatments in patients' homes.

\section{PROMISING MODEL FOR PAIN MANAGEMENT IN THE AFTERMATH OF THE OPIOID CRISIS}

The opioid crisis has clarified the need for a more holistic approach to pain management. With evidence of effectiveness for non-pharmacological options, there is growing momentum to broaden insurance coverage for these therapies. Like acupuncture, most non-pharmacological modalities share similar barriers to dissemination and implementation that may limit access and uptake despite greater insurance coverage. The new Medicare acupuncture policy, coupled with thoughtful attention to workforce training, interprofessional care coordination, and patient-centered care delivery, can provide a model to overcome these challenges and successfully integrate nonpharmacological therapies into pain management in a sustainable, equitable way.

Corresponding Author: Kevin T. Liou, MD; Bendheim Integrative Medicine Service, Department of Medicine, Memorial Sloan Kettering Cancer Center, New York, NY, USA (e-mail: liouk@mskcc.org).

Funding Information The writing of this manuscript was supported in part by a grant from the National Institutes of Health/National Cancer Institute (P3O CA008748) to Memorial Sloan Kettering Cancer Center.

\section{Compliance with Ethical Standards:}

Conflict of Interest: The spouse of Dr. Korenstein provided consulting services to Vedanta Biosciences and Takeda for work unrelated to the writing of this manuscript. Dr. Mao has received grants from Tibet Cheezheng Tibetan Medicine Co Ltd and Zhongke Health International LLC for work unrelated to the writing of this manuscript. Dr. Liou declares that he does not have a conflict of interest. 


\section{REFERENCES}

1. CMS finalizes decision to cover acupuncture for chronic low back pain for Medicare beneficiaries. The Centers for Medicare \& Medicaid Services. . https://www.cms.gov/newsroom/press-releases/cms-finalizes-decisioncover-acupuncture-chronic-low-back-pain-medicare-beneficiaries. Accessed January 21, 2020.

2. Gomes T, Tadrous M, Mamdani MM, Paterson JM, Juurlink DN. The burden of opioid-related mortality in the United States. JAMA Netw Open 2018;1(2):e180217.

3. Mao JJ, Davis RT, Coeytaux R, et al. Acupuncture for chronic low back pain: recommendations to Medicare/Medicaid from the Society for Acupuncture Research. J Altern Complement Med. 2019;25(4):367-369.

4. Fan AY, Stumpf SH, Faggert Alemi S, Matecki A. Distribution of licensed acupuncturists and educational institutions in the United States at the start of 2018. Complement Ther Med 2018;41:295-301.

5. Kuo YF, Raji MA, Chen NW, Hasan H, Goodwin JS. Trends in opioid prescriptions among Part D Medicare recipients from 2007 to 2012. Am J Med. 2016;129(2):221.e221-230.
6. Saper R. Integrative medicine and health disparities. Glob Adv Health Med 2016;5(1):5-8.

7. Liou KT, Hung TKW, Meghani SH, et al. What if acupuncture were covered by insurance for pain management? A cross-sectional study of cancer patients at one academic center and 11 community hospitals. Pain Med. 2019;20(10):2060-2068.

8. Niemtzow R, Baxter J, Gallagher RM, et al. Building capacity for complementary and integrative medicine through a large, cross-agency, acupuncture training program: lessons learned from a military health system and veterans health administration joint initiative project. Mil Med 2018;183(11-12):e486-e493.

Publisher's Note: Springer Nature remains neutral with regard to jurisdictional claims in published maps and institutional affiliations. 